\title{
Note on generalized Mittag-Leffler function
}

\author{
Rachana Desai ${ }^{1}$, I. A. Salehbhai ${ }^{2}$ and A. K. Shukla ${ }^{3^{*}}$
}

\section{*Correspondence:}

ajayshukla2@rediffmail.com

${ }^{3}$ Department of Applied

Mathematics \& Humanities,

S.V. National Institute

of Technology, Surat 395007,

India

Full list of author information

is available at the end of the article

\section{Abstract}

The present paper deals with the study of a generalized Mittag-Leffler function and associated fractional operator. The operator has been discussed in the space of Lebesgue measurable functions. The composition with Riemann-Liouville fractional integration operator has been obtained.

Keywords: Generalized Mittag-Leffler function, Fractional Calculus

Mathematics Subject Classification: 26A33, 33C20, 33E12

\section{Background}

The well-known Mittag-Leffler function $E_{\alpha}(z)$ named after its originator, the Swedish mathematician Gosta Mittag-Leffler (1846-1927), is defined by (Mittag-Leffler 1903)

$$
E_{\alpha}(z)=\sum_{n=0}^{\infty} \frac{z^{n}}{\Gamma(\alpha n+1)} ; \quad z \text { is a complex variable and } \operatorname{Re}(\alpha) \geq 0
$$

The Mittag-Leffler function naturally occurs as the solution of fractional order differential equations. The various generalization of Mittag-Leffler function have been defined and studied by different authors.

Shukla and Prajapati (2007) introduced its generalization $E_{\alpha, \beta}^{\gamma, q}(z)$, this is defined as

$$
E_{\alpha, \beta}^{\gamma, q}(z)=\sum_{n=0}^{\infty} \frac{(\gamma)_{q n}}{\Gamma(\alpha n+\beta)} \frac{z^{n}}{n !}
$$

for $\quad \alpha, \beta, \gamma \in C ; \quad \operatorname{Re}(\alpha)>0, \operatorname{Re}(\beta)>0, \operatorname{Re}(\gamma)>0, \operatorname{Re}(\delta)>0, \quad q \in(0,1) \cup N, \quad$ and $(\gamma)_{q n}=\frac{\Gamma(\gamma+q n)}{\Gamma(\gamma)}$ denotes the generalized Pochhammer symbol.

Further, the generalization of (2) is also given by Khan and Ahmed (2013), as follows:

$$
E_{\alpha, \beta, v, \sigma, \delta, p}^{\mu, \rho, \gamma, q}(z)=\sum_{n=0}^{\infty} \frac{(\mu)_{\rho n}(\gamma)_{q n} z^{n}}{\Gamma(\alpha n+\beta)(v)_{\sigma n}(\delta)_{p n}},
$$

where $\alpha, \beta, \gamma, \delta, \mu, v \in C ; p, q, \rho, \sigma>0 ; q \leq \operatorname{Re}(\alpha)+p ; \rho \leq \operatorname{Re}(\sigma)+p ; q \leq \operatorname{Re}(\sigma)+p$; $\rho, q \in(0,1) \cup N$ and $\min (\operatorname{Re}(\alpha), \operatorname{Re}(\beta), \operatorname{Re}(\gamma), \operatorname{Re}(\delta), \operatorname{Re}(\mu), \operatorname{Re}(v))>0$.

(C) 2016 The Author(s). This article is distributed under the terms of the Creative Commons Attribution 4.0 International License (http://creativecommons.org/licenses/by/4.0/), which permits unrestricted use, distribution, and reproduction in any medium, provided you give appropriate credit to the original author(s) and the source, provide a link to the Creative Commons license, and indicate if changes were made. 
Here, the convergence conditions of (3) have been modified, which was given by Khan and Ahmed (2013).

The following well-known notations and definitions have been used:

Let $L(a, b)$ (Kilbas et al. 2004) be a set of all Lebesgue measurable real or complex valued functions $f(x)$ on $[a, b]$ i.e.

$$
L(a, b)=\left\{f:\|f\|_{1} \equiv \int_{a}^{b}|f(t)| d t<\infty\right\}
$$

Let $f(x) \in L(a, b), \mu \in C(\operatorname{Re}(\mu)>0)$ then the Riemann-Liouville left-sided fractional integrals of order $\mu$ (Miller and Ross 1993) is defined as

$$
{ }_{a} I_{x}^{\mu} f(x)=I_{a+}^{\mu} f(x)=\frac{1}{\Gamma(\mu)} \int_{a}^{x} \frac{f(t)}{(x-t)^{1-\mu}} d t \quad(x>a)
$$

and the $\mathrm{R}-\mathrm{L}$ right-sided fractional integral of order $\mu$ is defined as

$$
{ }_{b} I_{x}^{\mu} f(x)=I_{b-}^{\mu} f(x)=\frac{1}{\Gamma(\mu)} \int_{x}^{b} \frac{f(t)}{(t-x)^{1-\mu}} d t \quad(x<b)
$$

Miller and Ross (1993) defined the following:

If $\mu, \alpha, \beta \in C, \operatorname{Re}(\mu)>0 ; n=[\operatorname{Re}(\mu)]+1 ; \operatorname{Re}(\beta)>0$ then

$$
I_{a+}^{\mu}\left[(t-a)^{\beta-1}\right](x)=\frac{\Gamma(\beta)}{\Gamma(\mu+\beta)}(x-a)^{\mu+\beta-1}
$$

and

$$
\left(D_{a+}^{\alpha} f\right)(x)=\left(\frac{d}{d x}\right)^{n}\left(I_{a+}^{n-\alpha} f\right)(x)
$$

Khan and Ahmed (2013) proved the following result.

If $\alpha, \beta, \gamma, \delta, \mu, v, \rho, \sigma \in C ; p, q>0 ; q \leq \operatorname{Re}(\alpha)+p$ and

$$
\operatorname{Re}(\alpha)>0, \operatorname{Re}(\beta)>0, \operatorname{Re}(\gamma)>0, \operatorname{Re}(\delta)>0, \operatorname{Re}(\mu)>0, \operatorname{Re}(v)>0, \operatorname{Re}(\rho)>0, \operatorname{Re}(\sigma)>0
$$

then for $m \in N$,

$$
\left(\frac{d}{d z}\right)^{m}\left[z^{\beta-1} E_{\alpha, \beta, v, \sigma, \delta, p}^{\mu, \rho, \gamma, q}\left(w z^{\alpha}\right)\right]=z^{\beta-m-1} E_{\alpha, \beta, v, \sigma, \delta, p}^{\mu, \rho, \gamma, q}\left(w z^{\alpha}\right) ; \quad \operatorname{Re}(\beta-m)>0 .
$$

In continuation of study, in this paper we give the operator associated with $E_{\alpha, \beta, v, \sigma, \delta, p}^{\mu, \rho, \gamma, q}(z)$ as follows:

Let $f(x) \in L(a, b)$, define

$$
\left(E_{\alpha, \beta, v, \sigma, \delta, p ; w ; a+}^{\mu, \rho, \gamma, q} f\right)(x)=\int_{a}^{x}(x-t)^{\beta-1} E_{\alpha, \beta, v, \sigma, \delta, p}^{\mu, \rho, \gamma, q}\left[w(x-t)^{\alpha}\right] f(t) d t ; \quad x>a,
$$


where $\alpha, \beta, \gamma, \delta, \mu, v \in C ; p, q, \rho, \sigma>0 ; q \leq \operatorname{Re}(\alpha)+p ; \rho \leq \operatorname{Re}(\sigma)+p ; q \leq \operatorname{Re}(\sigma)+p$ and $\min (\operatorname{Re}(\alpha), \operatorname{Re}(\beta), \operatorname{Re}(\gamma), \operatorname{Re}(\delta), \operatorname{Re}(\mu), \operatorname{Re}(v))>0$.

\section{Main results}

Using the definition (4), one can easily prove following lemma.

Lemma 1 If $\alpha, \beta, \gamma, \delta, \mu, v, \rho, \sigma \in C ; p, q>0 ; q \leq \operatorname{Re}(\alpha)+p ; x>a ; a \in R_{+}=[0, \infty)$ and $\min (\operatorname{Re}(\alpha), \operatorname{Re}(\beta), \operatorname{Re}(\gamma), \operatorname{Re}(\delta), \operatorname{Re}(\mu), \operatorname{Re}(v), \operatorname{Re}(\rho), \operatorname{Re}(\sigma))>0$, then

$$
E_{\alpha, \beta, v, \sigma, \delta, p}^{\mu, \rho, \gamma, q}\left(a t^{\alpha}\right)=\beta E_{\alpha, \beta+1, v, \sigma, \delta, p}^{\mu, \rho, \gamma, q}\left(a t^{\alpha}\right)+\alpha t E_{\alpha, \beta+1, v, \sigma, \delta, p}^{\mu, \rho, \gamma, q}\left(a t^{\alpha}\right)
$$

Theorem 1 Let $a \in R_{+}=[0, \infty)$. Let $\alpha, \beta, \gamma, \delta, \mu, v, \rho, \sigma \in C ; p, q>0 ; q \leq \operatorname{Re}(\alpha)+p$ and $\min (\operatorname{Re}(\alpha), \operatorname{Re}(\beta), \operatorname{Re}(\gamma), \operatorname{Re}(\delta), \operatorname{Re}(\mu), \operatorname{Re}(v), \operatorname{Re}(\rho), \operatorname{Re}(\sigma))>0, x>a$. Then

$$
\begin{aligned}
& \left(I_{a+}^{r}\left[(t-a)^{\beta-1} E_{\alpha, \beta, v, \sigma, \delta, p}^{\mu, \rho, \gamma, q}\left\{w(t-a)^{\alpha}\right\}\right)(x)=(x-a)^{r+\beta-1} E_{\alpha, \beta+r, v, \sigma, \delta, p}^{\mu, \rho, \gamma, q}\left[w(x-a)^{\alpha}\right]\right. \\
& \left(D_{a+[}^{r}\left[(t-a)^{\beta-1} E_{\alpha, \beta, v, \sigma, \delta, p}^{\mu, \rho, \gamma, q}\left\{w(t-a)^{\alpha}\right\}\right)(x)=(x-a)^{\beta-r-1} E_{\alpha, \beta-r, v, \sigma, \delta, p}^{\mu, \rho, \gamma, q}\left[w(x-a)^{\alpha}\right]\right.
\end{aligned}
$$

Proof Using definitions (3) and (5) and further simplification gives

$$
\begin{aligned}
\left(I_{a+}^{r}\right. & {\left[(t-a)^{\beta-1} E_{\alpha, \beta, v, \sigma, \delta, p}^{\mu, \gamma, q}\left\{w(t-a)^{\alpha}\right\}\right)(x) } \\
& =\sum_{n=0}^{\infty} \frac{(\mu)_{\rho n}(\gamma)_{q n}}{\Gamma(\alpha n+\beta)} \frac{w^{n}}{(v)_{\sigma n}(\delta)_{p n}}\left(I_{a+}^{r}\left[(t-a)^{\alpha n+\beta-1}\right]\right)(x) \\
& =\sum_{n=0}^{\infty} \frac{(\mu)_{\rho n}(\gamma)_{q n}}{\Gamma(\alpha n+\beta)} \frac{w^{n}}{(v)_{\sigma n}(\delta)_{p n}} \frac{\Gamma(\alpha n+\beta)}{\Gamma(\alpha n+\beta+r)}(x-a)^{\alpha n+\beta+r-1} \\
& =(x-a)^{r+\beta-1} \sum_{n=0}^{\infty} \frac{(\mu)_{\rho n}(\gamma)_{q n}}{\Gamma(\alpha n+\beta+r)} \frac{\left[w(x-a)^{\alpha}\right]^{n}}{(v)_{\sigma n}(\delta)_{p n}} \\
& =(x-a)^{r+\beta-1} E_{\alpha, \beta+r, v, \sigma, \delta, p}^{\mu, \rho, \gamma, q}\left[w(x-a)^{\alpha}\right] .
\end{aligned}
$$

This completes the proof of (12).

To prove (13), we use definitions (8) and further simplification gives

$$
\begin{aligned}
& \left(D_{a+}^{r}\left[(t-a)^{\beta-1} E_{\alpha, \beta, v, \sigma, \delta, p}^{\mu, \rho, \gamma, q}\left\{w(t-a)^{\alpha}\right\}\right)(x)\right. \\
& \quad=\left(\frac{d}{d x}\right)^{n}\left(I_{a+}^{n-r}\left[(t-a)^{\beta-1} E_{\alpha, \beta, v, \sigma, \delta, p}^{\mu, \rho, \gamma, q}\left\{w(t-a)^{\alpha}\right\}\right]\right)(x),
\end{aligned}
$$

On applying (12) with replacement of $r$ by $n-r$, the above equation reduces to

$$
=\left(\frac{d}{d x}\right)^{n}\left[(x-a)^{\beta+n-r-1} E_{\alpha, \beta+n-r, v, \sigma, \delta, p}^{\mu, \rho, \gamma, q}\left\{w(x-a)^{\alpha}\right\}\right],
$$


From (9), we get

$$
\begin{aligned}
& =(x-a)^{\beta+n-r-1-n} E_{\alpha, \beta+n-r-n, v, \sigma, \delta, p}^{\mu, \rho, \gamma, q}\left\{w(x-a)^{\alpha}\right\} \\
& =(x-a)^{\beta-r-1} E_{\alpha, \beta-r, v, \sigma, \delta, p}^{\mu, \rho, q}\left[w(x-a)^{\alpha}\right] .
\end{aligned}
$$

Theorem 2 Let $a \in R_{+}=[0, \infty), \alpha, \beta, \gamma, \delta, \mu, v, \rho, \sigma \in C ; p, q>0$ and $q \leq \operatorname{Re}(\alpha)+p$ and $\min (\operatorname{Re}(\alpha), \operatorname{Re}(\beta), \operatorname{Re}(\gamma), \operatorname{Re}(\delta), \operatorname{Re}(\mu), \operatorname{Re}(v), \operatorname{Re}(\rho), \operatorname{Re}(\sigma))>0 x>a$. Then

$$
\left(E_{\alpha, \beta, v, \sigma, \delta, p ; w ; a+}^{\mu, \rho, \gamma, q}(t-a)^{r-1}\right)(x)=(x-a)^{\beta+r-1} \Gamma(r) E_{\alpha, \beta+r, v, \sigma, \delta, p}^{\mu, \rho, \gamma, q}\left(w(x-a)^{\alpha}\right) .
$$

Proof Taking $f(t)=(t-a)^{r-1}$ in (10), we get

$$
\begin{aligned}
& \left(E_{\alpha, \beta, v, \sigma, \delta, p ; w ; a+}^{\mu, \rho, \gamma, q}(t-a)^{r-1}\right)(x) \\
& =\int_{a}^{x}(x-t)^{\beta-1} E_{\alpha, \beta, v, \sigma, \delta, p}^{\mu, \rho, \gamma, q}\left[w(x-t)^{\alpha}\right](t-a)^{r-1} d t \\
& =\sum_{n=0}^{\infty} \frac{(\mu)_{\rho n}(\gamma)_{q n}}{\Gamma(\alpha n+\beta)} \frac{w^{n}}{(v)_{\sigma n}(\delta)_{p n}} \int_{a}^{x}(x-t)^{\alpha n+\beta-1}(t-a)^{r-1} d t,
\end{aligned}
$$

Replacing $t$ by $a+(x-a) t$ and simplifying the above equation

$$
=\sum_{n=0}^{\infty} \frac{(\mu)_{\rho n}(\gamma)_{q n}}{\Gamma(\alpha n+\beta)} \frac{w^{n}}{(v)_{\sigma n}(\delta)_{p n}}(x-a)^{\alpha n+\beta+r-1} B(\alpha n+\beta, r)
$$

and further simplification of above equation gives the proof of Theorem 2 .

Theorem 3 Let $a \in R_{+}=[0, \infty), \alpha, \beta, \gamma, \delta, \mu, v, \rho, \sigma \in C ; p, q>0$ and $q \leq \operatorname{Re}(\alpha)+p$ and $\min (\operatorname{Re}(\alpha), \operatorname{Re}(\beta), \operatorname{Re}(\gamma), \operatorname{Re}(\delta), \operatorname{Re}(\mu), \operatorname{Re}(v), \operatorname{Re}(\rho), \operatorname{Re}(\sigma))>0, b>a$., Then the operator $E_{\alpha, \beta, v, \sigma, \delta, p ; w ; a+}^{\mu, \rho, \gamma}$ is bounded on $L(a, b)$ and

$$
\left\|E_{\alpha, \beta, v, \sigma, \delta, p ; w ; a+}^{\mu, \rho, \gamma} f\right\|_{1} \leq B\|f\|_{1}
$$

where

$$
B=(b-a)^{\operatorname{Re}(\beta)} \sum_{n=0}^{\infty} \frac{\left|(\mu)_{\rho n}\right|\left|(\gamma)_{q n}\right|}{[\operatorname{Re}(\alpha) n+\operatorname{Re}(\beta)]|\Gamma(\alpha n+\beta)|} \frac{\left|w(b-a)^{\operatorname{Re}(\alpha)}\right|^{n}}{\left|(v)_{\sigma n}\right|\left|(\delta)_{p n}\right|} .
$$

Proof On using the definition (10) and applying Dirichlet's formula (Samko et al. 1993), we have

$$
\begin{aligned}
\left\|E_{\alpha, \beta, v, \sigma, \delta, p ; w ; a+}^{\mu, \rho, \gamma, q} f\right\|_{1} & =\int_{a}^{b}\left|\int_{a}^{x}(x-t)^{\beta-1} E_{\alpha, \beta, v, \sigma, \delta, p}^{\mu, \rho, \gamma, q}\left[w(x-t)^{\alpha}\right] f(t) d t\right| d x \\
& \leq \int_{a}^{b}\left[\int_{t}^{b}(x-t)^{\operatorname{Re}(\beta)-1}\left|E_{\alpha, \beta, v, \sigma, \delta, p}^{\mu, \rho, \gamma, q}\left[w(x-t)^{\alpha}\right]\right| d x\right]|f(t)| d t,
\end{aligned}
$$


Taking $u=x-t$ in inner integral, this yields

$$
\begin{aligned}
& \left\|E_{\alpha, \beta, v, \sigma, \delta, p ; w ; a+}^{\mu, \rho, \gamma, q} f\right\|_{1} \\
& \quad \leq \int_{a}^{b}\left[\int_{0}^{b-t} u^{\operatorname{Re}(\beta)-1}\left|E_{\alpha, \beta, v, \sigma, \delta, p}^{\mu, \rho, \gamma, q}\left[w u^{\alpha}\right]\right| d u\right]|f(t)| d t \\
& \leq \int_{a}^{b} \sum_{n=0}^{\infty} \frac{\left|(\mu)_{\rho n}\right|\left|(\gamma)_{q n}\right|}{|\Gamma(\alpha n+\beta)|} \frac{|w|^{n}}{\left|(v)_{\sigma n}\right|\left|(\delta)_{p n}\right|}\left[\frac{u^{\operatorname{Re}(\alpha) n+\operatorname{Re}(\beta)}}{\operatorname{Re}(\alpha) n+\operatorname{Re}(\beta)}\right]_{0}^{b-a}|f(t)| d t \\
& \quad=(b-a)^{\operatorname{Re}(\beta)} \sum_{n=0}^{\infty} \frac{\left|(\mu)_{\rho n}\right|\left|(\gamma)_{q n}\right|}{|\Gamma(\alpha n+\beta)|} \frac{|w|^{n}}{\left|(v)_{\sigma n}\right|\left|(\delta)_{p n}\right|} \frac{(b-a)^{\operatorname{Re}(\alpha) n}}{[\operatorname{Re}(\alpha) n+\operatorname{Re}(\beta)]} \int_{a}^{b}|f(t)| d t
\end{aligned}
$$

This completes the proof.

Theorem 4 (Composition with Riemann-Liouville fractional integration operator) Let $\alpha, \beta, \gamma, \delta, \mu, v, \rho, \sigma \in C ; p, q>0 ; q \leq \operatorname{Re}(\alpha)+p ; b>a$ and $\min (\operatorname{Re}(\alpha), \operatorname{Re}(\beta), \operatorname{Re}(\gamma)$, $\operatorname{Re}(\delta), \operatorname{Re}(\mu), \operatorname{Re}(v), \operatorname{Re}(\rho), \operatorname{Re}(\sigma))>0$. Then the relation

$$
I_{a+}^{r} E_{\alpha, \beta, v, \sigma, \delta, p ; w ; a+}^{\mu, \rho, \gamma, q} f \equiv E_{\alpha, \beta+r, v, \sigma, \delta, p ; w ; a+}^{\mu, \rho, \gamma, q} f \equiv E_{\alpha, \beta, v, \sigma, \delta, p ; w ; a+}^{\mu, \rho, \gamma, q} I_{a+}^{r} f
$$

holds for any summable function $f \in L(a, b)$.

Proof From (10) and (5), we get

$$
\left(I_{a+}^{r} E_{\alpha, \beta, v, \sigma, \delta, p ; w ; a+}^{\mu, \rho, \gamma, q} f\right)(x)=\frac{1}{\Gamma(r)} \int_{a}^{x} \int_{a}^{u}(x-u)^{r-1}(u-t)^{\beta-1} E_{\alpha, \beta, v, \sigma, \delta, p}^{\mu, \rho, \gamma, q}\left[w(u-t)^{\alpha}\right] f(t) d t d u
$$

Applying Dirichlet's formula (Samko et al. 1993), we get

$$
=\int_{a}^{x}\left[\frac{1}{\Gamma(r)} \int_{t}^{x}(x-u)^{r-1}(u-t)^{\beta-1} E_{\alpha, \beta, v, \sigma, \delta, p}^{\mu, \rho, \gamma, q}\left[w(u-t)^{\alpha}\right] d u\right] f(t) d t
$$

Substituting $u-t=\tau$ in the above equation, we get

$$
=\int_{a}^{x}\left[\frac{1}{\Gamma(r)} \int_{0}^{x-t}(x-t-\tau)^{r-1} \tau^{\beta-1} E_{\alpha, \beta, v, \sigma, \delta, p}^{\mu, \rho, \gamma, q}\left[w \tau^{\alpha}\right] d \tau\right] f(t) d t
$$

Again using (5), this equation becomes

$$
=\int_{a}^{x}\left(I_{0+}^{\mu}\left[\tau^{\beta-1} E_{\alpha, \beta}^{\gamma, q}\left(w \tau^{\alpha}\right)\right]\right)(x-t) f(t) d t
$$


Applying (12), this yields

$$
=\int_{a}^{x}(x-t)^{\beta+r-1} E_{\alpha, \beta+r, v, \sigma, \delta, p}^{\mu, \rho, \gamma, q}\left[w(x-t)^{\alpha}\right] f(t) d t,
$$

Using (10), we get

$$
=\left(E_{\alpha, \beta+r, v, \sigma, \delta, p ; w ; a+}^{\mu, \rho, \gamma, q} f(x) .\right.
$$

The other equality can also be proved in the similar way.

Theorem 5 Let $\alpha, \beta, \gamma, \delta, \mu, v, \rho, \sigma \in C ; p, q>0 ; q \leq \operatorname{Re}(\alpha)+p ; b>a$ and $\min (\operatorname{Re}(\alpha)$, $\operatorname{Re}(\beta), \operatorname{Re}(\gamma), \operatorname{Re}(\delta), \operatorname{Re}(\mu), \operatorname{Re}(v), \operatorname{Re}(\rho), \operatorname{Re}(\sigma))>0$. Then the relation

$$
D_{a+}^{r} E_{\alpha, \beta, v, \sigma, \delta, p ; w ; a+}^{\mu, \rho, \gamma} f \equiv E_{\alpha, \beta-r, v, \sigma, \delta, p ; w ; a+}^{\mu, \rho, \gamma} f
$$

holds for any continuous function $f \in C[a, b]$.

Proof From (8), we have

$$
\left(D_{a+}^{r} E_{\alpha, \beta, v, \sigma, \delta, p ; w ; a+}^{\mu, \rho, \gamma, q} f\right)(x)=\left(\frac{d}{d x}\right)^{n}\left(I_{a+}^{n-r} E_{\alpha, \beta, v, \sigma, \delta, p ; w ; a+}^{\mu, \rho, \gamma} f\right)(x)
$$

Again using Theorem 4 and definition (10),

$$
=\left(\frac{d}{d x}\right)^{n}\left[\int_{a}^{x}(x-t)^{\beta+n-r-1} E_{\alpha, \beta+n-r, v, \sigma, \delta, p}^{\mu, \rho, \gamma, q}\left[w(x-t)^{\alpha}\right] f(t) d t\right]
$$

The integrand in the above equation is continuous function on $[a, b]$, here we take

$$
\frac{d}{d x} \int_{a}^{x} h(x, t) d t=\int_{a}^{x} \frac{\partial}{\partial x} h(x, t) d t+h(x, x)
$$

From (19) and (20), we get

$$
\left(D_{a+}^{r} E_{\alpha, \beta, v, \sigma, \delta, p ; w ; a+}^{\mu, \rho, \gamma, q} f\right)(x)=\left(\frac{d}{d x}\right)^{n-1} \int_{a}^{x}(x-t)^{\beta+n-r-2} E_{\alpha, \beta+n-r-1, v, \sigma, \delta, p}^{\mu, \rho, \gamma, q}\left[w(x-t)^{\alpha}\right] f(t) d t
$$

Applying same procedures as above, this led the proof of the theorem. This is easy to prove by using mathematical induction method also.

Theorem 6 Let $a \in R_{+}=[0, \infty), \alpha, \beta, \gamma, \delta, \mu, v, \rho, \sigma \in C ; p, q>0 ; q \leq \operatorname{Re}(\alpha)+p$ and $\min (\operatorname{Re}(\alpha), \operatorname{Re}(\beta), \operatorname{Re}(\gamma), \operatorname{Re}(\delta), \operatorname{Re}(\mu), \operatorname{Re}(v), \operatorname{Re}(\rho), \operatorname{Re}(\sigma))>0, \quad x>a$. Then

$$
\left(I_{0+}^{r}\left[t^{\beta-1} E_{\alpha, \beta, v, \sigma, \delta, p}^{\mu, \rho, \gamma, q}\left\{a t^{\alpha}\right\}\right)(x)=x^{r+\beta-1} E_{\alpha, \beta+r, v, \sigma, \delta, p}^{\mu, \rho, \gamma, q}\left[a x^{\alpha}\right]\right.
$$


Proof We have

$$
\begin{aligned}
\left(I_{0+}^{r}\left[t^{\beta-1} E_{\alpha, \beta, v, \sigma, \delta, p}^{\mu, \rho, \gamma, q}\left\{a t^{\alpha}\right\}\right)(x)\right. & =\sum_{n=0}^{\infty} \frac{(\mu)_{\rho n}(\gamma)_{q n}}{\Gamma(\alpha n+\beta)} \frac{a^{n}}{(v)_{\sigma n}(\delta)_{p n}}\left(I_{0+}^{r}\left[t^{\alpha n+\beta-1}\right]\right)(x) \\
& =\sum_{n=0}^{\infty} \frac{(\mu)_{\rho n}(\gamma)_{q n}}{\Gamma(\alpha n+\beta)} \frac{a^{n}}{(v)_{\sigma n}(\delta)_{p n}} \frac{\Gamma(\alpha n+\beta)}{\Gamma(\alpha n+\beta+r)}(x)^{\alpha n+\beta+r-1} \\
& =x^{r+\beta-1} \sum_{n=0}^{\infty} \frac{(\mu)_{\rho n}(\gamma)_{q n}}{\Gamma(\alpha n+\beta+\mu)} \frac{\left[a x^{\alpha}\right]^{n}}{(v)_{\sigma n}(\delta)_{p n}} \\
& =x^{r+\beta-1} E_{\alpha, \beta+r, v, \sigma, \delta, p}^{\mu, \rho, \gamma, q}\left[a x^{\alpha}\right] .
\end{aligned}
$$

This completes the proof.

Corollary 1 If $\alpha, \beta, \gamma, \delta, \mu, v, \rho, \sigma \in C ; \quad p, q>0 \quad$ and $\quad q \leq \operatorname{Re}(\alpha)+p x>a$; $\min (\operatorname{Re}(\alpha), \operatorname{Re}(\beta), \operatorname{Re}(\gamma), \operatorname{Re}(\delta), \operatorname{Re}(\mu), \operatorname{Re}(v), \operatorname{Re}(\rho), \operatorname{Re}(\sigma))>0, \quad a \in R_{+}=[0, \infty)$. Let $I_{0+}^{r}$ be the left-sided operator of Riemann-Liouville fractional integral. Then

$$
\left(I_{0+}^{r}\left[t^{\beta-1} E_{\alpha, \beta, v, \sigma, \delta, p}^{\mu, \rho, \gamma, q}\left\{a t^{\alpha}\right\}\right)(x)=x^{r+\beta-1}\left\{(\beta+r) E_{\alpha, \beta+r+1, v, \sigma, \delta, p}^{\mu, \rho, \gamma, q}+x \frac{d}{d x} E_{\alpha, \beta+r+1, v, \sigma, \delta, p}^{\mu, \rho, \gamma, q}\left(a x^{\alpha}\right)\right\}\right.
$$

Proof is very obvious from Lemma 1 and Theorem 6.

Theorem 7 Let $a \in R_{+}=[0, \infty), \alpha, \beta, \gamma, \delta, \mu, v, \rho, \sigma \in C ; p, q>0 ; q \leq \operatorname{Re}(\alpha)+p$ and $\min (\operatorname{Re}(\alpha), \operatorname{Re}(\beta), \operatorname{Re}(\gamma), \operatorname{Re}(\delta), \operatorname{Re}(\mu), \operatorname{Re}(v), \operatorname{Re}(\rho), \operatorname{Re}(\sigma))>0, x>a, I_{-}^{r}$ be the rightsided operator of Riemann-Liouville fractional integral. Then

$$
\left(I_{-}^{r}\left[t^{-r-\beta} E_{\alpha, \beta, v, \sigma, \delta, p}^{\mu, \rho, \gamma, q}\left\{a t^{-\alpha}\right\}\right)(x)=x^{-\beta} E_{\alpha, \beta+r, v, \sigma, \delta, p}^{\mu, \rho, \gamma}\left[a x^{-\alpha}\right]\right.
$$

Proof Let

$$
\left(I_{-}^{r}\left[t^{-r-\beta} E_{\alpha, \beta, v, \sigma, \delta, p}^{\mu, \rho, \gamma, q}\left\{a t^{-\alpha}\right\}\right)(x)=\frac{1}{\Gamma(r)} \int_{x}^{\infty}(t-x)^{r-1} t^{-r-\beta} \sum_{n=0}^{\infty} \frac{(\mu)_{\rho n}(\gamma)_{q n}\left(a t^{-\alpha}\right)^{n}}{\Gamma(\alpha n+\beta)(v)_{\sigma n}(\delta)_{p n}} d t\right.
$$

On changing the order of the summation and integration then afterward applying beta function, this gives

$$
=x^{-\beta} \sum_{n=0}^{\infty} \frac{(\mu)_{\rho n}(\gamma)_{q n}\left(a x^{-\alpha}\right)^{n}}{\Gamma(\alpha n+\beta+r)(v)_{\sigma n}(\delta)_{p n}}=x^{-\beta} E_{\alpha, \beta+r, v, \sigma, \delta, p}^{\mu, \rho, \gamma}\left\{a x^{-\alpha}\right\}
$$

Corollary 2 If $\alpha, \beta, \gamma, \delta, \mu, v, \rho, \sigma \in C ; \quad p, q>0$ and $q \leq \operatorname{Re}(\alpha)+p ; \quad x>a$; $\min (\operatorname{Re}(\alpha), \operatorname{Re}(\beta), \operatorname{Re}(\gamma), \operatorname{Re}(\delta), \operatorname{Re}(\mu), \operatorname{Re}(v), \operatorname{Re}(\rho), \operatorname{Re}(\sigma))>0, a \in R_{+}=[0, \infty)$. Let $I_{-}^{r}$ be the right-sided operator of Riemann-Liouville fractional integral. Then

$$
\left(I_{-}^{r}\left[t^{-r-\beta} E_{\alpha, \beta, v, \sigma, \delta, p}^{\mu, \rho, \gamma, q}\left\{a t^{-\alpha}\right\}\right)(x)=x^{-r-\beta}\left\{(\beta+r) E_{\alpha, \beta+r+1, v, \sigma, \delta, p}^{\mu, \rho, \gamma, q}+x \frac{d}{d x} E_{\alpha, \beta+r+1, v, \sigma, \delta, p}^{\mu, \rho, \gamma, q}\left(a x^{-\alpha}\right)\right\}\right.
$$




\section{Conclusion}

In this paper, we proved some properties of generalized Mittag-Leffler functions and also used the fractional calculus approach to prove Theorems 4, 5, 6 and 7.

\section{Authors' contributions}

The authors contributed equally and significantly in writing this article. All authors read and approved the final manuscript.

\section{Author details}

${ }^{1}$ Department of Mathematics, K. J. Somaiya College of Engineering, Vidyavihar, Mumbai 400077, India. ${ }^{2}$ Department of Mathematics, Government Engineering College, Bharuch 392002, India. ${ }^{3}$ Department of Applied Mathematics \& Humanities, S.V. National Institute of Technology, Surat 395007, India.

\section{Competing interests}

The authors declare that they have no competing interests.

Received: 16 September 2015 Accepted: 6 May 2016

Published online: 21 May 2016

\section{References}

Khan MA, Ahmed S (2013) On some properties of the generalized Mittag-Leffler function. SpringerPlus 2:1-9

Kilbas AA, Sagio M, Saxena RK (2004) Generalized Mittag-Leffler function and generalized fractional calculus operators. Integral Trans Spec Funct 15(1):31-49

Miller KS, Ross B (1993) An introduction to fractional calculus and fractional differential equations. Wiley, New York Mittag- Leffler G (1903) Sur la nouvelle function $E_{a}(x)$. C R Acad Sci Paris 137:554-558

Samko SG, Kilbas AA, Marichev OI (1993) Fractional integrals and derivatives, theory and applications. Gordon and Breach, New York

Shukla AK, Prajapati JC (2007) On a generalization of Mittag-Leffler function and its properties. J Math Anal Appl 336(2):797-811

\section{Submit your manuscript to a SpringerOpen ${ }^{\circ}$ journal and benefit from:}

- Convenient online submission

Rigorous peer review

- Immediate publication on acceptance

- Open access: articles freely available online

- High visibility within the field

Retaining the copyright to your article

Submit your next manuscript at $>$ springeropen.com 